\title{
Effects of arginine on intestinal epithelial cell integrity and nutrient uptake
}

\author{
Mi Xia†, Lulu Ye†, Qihang Hou and Qinghua Yu* \\ Veterinary College, Nanjing Agricultural University, Weigang 1, Nanjing, Jiangsu 210095, People's Republic of China \\ (Submitted 20 May 2016 - Final revision received 27 August 2016 - Accepted 10 October 2016 - First published online 14 November 2016)
}

\begin{abstract}
Arginine is a multifaceted amino acid that is critical to the normal physiology of the gastrointestinal tract. Oral arginine administration has been shown to improve mucosal recovery following intestinal injury. The present study investigated the influence of extracellular arginine concentrations on epithelial cell barrier regulation and nutrition uptake by porcine small intestinal epithelial cell line (IPEC-J2). The results show that reducing arginine concentration from 0.7 to $0.2 \mathrm{~mm}$ did not affect the transepithelial electrical resistance value, tight-junction proteins (claudin-1, occludin, E-cadherin), phosphorylated extracellular signal-regulated protein kinases (p-ERK) and mucin-1 expression. Furthermore, reducing arginine concentration stimulated greater expression of cationic amino acid transporter (CAT1), excitatory amino acid transporter (EAAT3) and alanine/ serine/cysteine transporter (ASCT1) mRNA by IPEC-J2 cells, which was verified by elevated efficiency of amino acid uptake. Glucose consumption by IPEC-J2 cells treated with $0.2 \mathrm{~mm}$-arginine remained at the same physiological level to guarantee energy supply and to maintain the cell barrier. This experiment implied that reducing arginine concentration is feasible in IPEC-J2 cells guaranteed by nutrient uptake and cell barrier function.
\end{abstract}

\section{Key words: Arginine: Intestinal epithelium: Mucosal barrier: Nutrient absorption}

The intestinal mucosal epithelium acts as a physical barrier to pathogens ${ }^{(1)}$. The mucosal barrier is composed primarily of mucus and an epithelial cell membrane ${ }^{(2)}$. Changes to the mucosal barrier can affect the absorption of both exogenous and endogenous nutrients ${ }^{(3)}$. Therefore, the integrity of the mucosal barrier plays a crucial role in nutrition. Porcine small intestinal epithelial cell line (IPEC-J2) is an intestinal columnar epithelial cell line that was isolated from the mid-jejunum of a neonatal piglet. The IPEC-J2 cell line provides researchers with a unique tool: specificity for swine-based diseases and nutritional studies. Given the high homology of porcine and human intestinal structure and function and presumably similarity of enterocyte structure and function, studies using IPEC-J2 cells may provide valuable insights to the interaction between nutrients and the human intestinal mucosa ${ }^{(4)}$.

Arginine is an essential amino acid in neonates, synthesised by enterocytes and required for protein synthesis and maximum growth $^{(5,6)}$. It is the precursor of many important biological molecules such as proteins, nitric oxide (NO), ornithine and polyamine. Arginine signalling is important in the intestine, as it improves the structure and function of the intestinal mucosa ${ }^{(7)}$. Dietary arginine also promotes the growth of piglets, as well as the development of intestine $^{(8)}$. More recently, it has been shown that supplemental arginine administration before a wound injury in IPEC-J2 cells could increase cell migration in a dose-dependent biphasic manner via NO and focal adhesion kinase-dependent mechanisms ${ }^{(9,10)}$. In addition, a recent study demonstrated that arginine treatment increased protein synthesis in IPEC-J2 cells via the rapamycin (mTOR)/ribosomal protein S6 kinase (p70S6K) pathway ${ }^{(11)}$.

Most published studies focus on the effect of arginine on growth performance. However, little is known about the effect of the concentration of arginine on the intestinal epithelial cell barrier and nutrition uptake in vitro, given that more amino acid than necessary will cause waste and increase environment pressure, and highquality protein is short in China. In the present study, we aimed to explore the influence of reduction of arginine concentration $(0$ and $0 \cdot 2 \mathrm{~mm}$ ) in IPEC-J2 on cell integrity, as well as nutrient absorption.

\section{Methods}

\section{Cell culture}

In brief, IPEC-J2 cells were seeded on collagen-coated Millicell filter inserts with a $0 \cdot 4-\mu \mathrm{m}$ pore size (Corning). IPEC-J2 cells were cultured and maintained in Dulbecco's modified Eagle's medium/nutrient mixture F-12 (DMEM-F12) (Life) supplemented with $10 \%$ fetal bovine serum (Gibco) and penicillin-streptomycin $(100 \mathrm{mg} / \mathrm{ml}$; Invitrogen). When the IPEC-J2 cells reached confluence (transepithelial electrical resistance (TEER) $\left.\geq 1000 \Omega \times \mathrm{cm}^{2}\right)^{(12)}$, cell culture medium was changed to new medium supplemented with arginine at varying concentrations (arginine-free, 0.2 or $0.7 \mathrm{~mm}$-arginine).

\footnotetext{
Abbreviations: ASCT1, alanine/serine/cysteine transporter; CAT1, cationic amino acid transporter; EAAT3, excitatory amino acid transporter; GLUT2, glucose transporter type 2; IPEC-J2, small intestinal epithelial cell line; p-ERK, phosphorylated extracellular signal-regulated protein kinases; SGLT1, sodium/glucose cotransporter 1; Slc, solute carrier; TEER, transepithelial electrical resistance; TJ, tight junction.
}

* Corresponding author: Q. Yu, fax +86 2584398669, email yuqinghua1981@163.com

$\dagger$ These authors contributed equally to this work. 


\section{Transepithelial electrical resistance assay}

TEER, a measure of tight-junction (TJ) integrity, is measured by an epithelial tissue volt-ohmmeter (Millicell ERS-2; Millipore Corporation) ${ }^{(13)}$. TEER was calculated from the measured potential difference between the apical and basolateral sides of the cell layer by an epithelial tissue volt-ohmmeter. All cells were measured in real time (on the 3rd, 5th, 7th, 9th, 11th and 12th day). Resistance is expressed as $\Omega \times \mathrm{cm}^{2}$.

\section{Western blot analysis}

The IPEC-J2 cells were collected and lysed in radioimmunoprecipitation assay (RIPA) lysis buffer (SunShine Bio). After centrifugation at $10000 \mathbf{g}$ and $4^{\circ} \mathrm{C}$ for $10 \mathrm{~min}$, the protein concentration in the supernatant was determined using a Tecan Infinite 200 Pro (Tecan). All samples were adjusted to the same protein concentration, diluted in $4 \times$ loading buffer to a final volume of $2.5 \mathrm{ml}$ and heated in boiling water for $10 \mathrm{~min}$. After cooling on ice, the solution was used for Western blot analysis.

Equal amounts of sample aliquots were subjected to $12 \%$ SDS-PAGE and transferred to polyvinylidene difluoride (PVDF) membranes (Millipore) at $90 \mathrm{~V}$ for 100 min using the Bio-Rad Transblot apparatus (Trans-blot Turbo system; Bio-Rad). The membranes were blocked in 5\% fat-free milk in TBST (Trisbuffered saline, $0.5 \%$ Tween 20 ) for $2 \mathrm{~h}$ and were then incubated overnight with sodium/glucose cotransporter 1 (SGLT1) (Santa Cruz; 1:800), glucose transporter type 2 (GLUT2) (Santa Cruz; 1:1000), claudin-1 (Santa Cruz; 1:1000), occludin (Abcam; 1:1000), E-cadherin (Abcam; 1:500), phosphorylated extracellular signal-regulated protein kinases (p-ERK) (Bioworld Technology; 1:1000), tubulin (Abcam; 1:1000) or $\beta$-actin (Vazyme Biotech; 1:5000) antibodies at $4^{\circ} \mathrm{C}$ with gentle rocking.

After washing five times with TBST, the membranes were incubated at room temperature for $2 \mathrm{~h}$ with horseradishperoxidase-linked secondary antibody (Vazyme Biotech; 1:6000). Finally, the membranes were washed with TBST and then developed using Supersignal West Dura Extended Duration Substrate (Pierce) according to the manufacturer's instructions. The images were detected by chemiluminescence (Applygen). Western blots were quantified by measuring the intensity of the correctly sized bands using Quantity One-4.6.2 (Bio-Rad). The ratio of the intensities of the protein bands of interest $v$. the housekeeping protein was calculated for each filter, and the ratios from different Western blot filters were used to determine protein abundance.

\section{Extraction of RNA and gene expression analysis in the jejunum using real-time $P C R$}

Total RNA of IPEC-J2 cells was extracted from the cells using Trizol reagent (Invitrogen). RNA integrity was assessed by $1 \%$ agarose gel electrophoresis and staining with $10 \mathrm{mg} / \mathrm{ml}$ ethidium bromide. First-strand complementary DNA synthesis was performed with the quantitative reverse transcription (QRT) reverse transcriptase (Vazyme Biotech). Real-time PCR was conducted using the 7500 Real-Time PCR System (ABI) and EvaGreen qPCR MasterMix-Low Rox (Abm). PCR amplification
Table 1. Primers used for real-time PCR

\begin{tabular}{|c|c|c|c|c|}
\hline Genes & Primers & Sequences $\left(5^{\prime}-3^{\prime}\right)^{*}$ & Size (bp) & $\operatorname{Tm}\left({ }^{\circ} \mathrm{C}\right)$ \\
\hline \multirow[t]{2}{*}{$\beta$-Actin } & Forward & AGATCAAGATCATCGCGCCT & \multirow[t]{2}{*}{171} & \multirow[t]{2}{*}{60} \\
\hline & Reverse & ATGCAACTAACAGTCCGCCT & & \\
\hline \multirow[t]{2}{*}{ Slc1A1 } & Forward & CCAGCAGTACAAAACCACGC & \multirow[t]{2}{*}{167} & \multirow[t]{2}{*}{59} \\
\hline & Reverse & AGCCCAGGACATTTATGCCG & & \\
\hline \multirow[t]{2}{*}{ Slc1A4 } & Forward & TTCATTCTTCCCATCGGGGC & \multirow[t]{2}{*}{138} & \multirow[t]{2}{*}{60} \\
\hline & Reverse & CGTGGCTGTCACTAGGATGG & & \\
\hline \multirow[t]{2}{*}{ Slc7A1 } & Forward & TCTGAACGTCCGTCAGTTCG & \multirow[t]{2}{*}{134} & \multirow[t]{2}{*}{60} \\
\hline & Reverse & GGAGGAGGGTCCTGTGATCT & & \\
\hline
\end{tabular}

Tm, melting temperature; SIC1A1, solute carrier family 1 member 1 ; SIC1A4, solute carrier family 1 member 4 ; SIC7A1, solute carrier family 7 member 1 ; NCBI, National Center for Biotechnology Information.

* Sequences from the NCBI database.

was conducting using the following conditions: $95^{\circ} \mathrm{C}$ for $10 \mathrm{~min}$, followed by thirty-five cycles of $95^{\circ} \mathrm{C}$ for $30 \mathrm{~s}$ and $60 \mathrm{C}$ for $34 \mathrm{~s}$. Each sample and negative controls (no template) were run in triplicate. The data were analysed using equation $2^{-\Delta \Delta C_{T}}$. The mRNA expression of solute carrier family 1 member 4 (Slc1A4) (alanine/serine/cysteine transporter; ASCT1), solute carrier family 1 member 1 (Slc1A1) (excitatory amino acid transporter; $E A A T 3$ ) and solute carrier family 7 member 1 (Slc 7A1) (cationic amino acid transporter; CAT1) was analysed. $\beta$-Actin was used as a housekeeping gene for data normalisation (Table 1).

Amino acid detection in cell culture medium using L-8900, a highly efficient amino acid analyzer

The amount of amino acids made by IPEC-J2 cells was measured after 12 or $24 \mathrm{~h}$ of incubation in a glucose production medium ( $\mathrm{pH} 7.4$ ) supplemented with different arginine concentrations. For each sample, $200 \mu \mathrm{l}$ of medium was deproteinised with $200 \mu \mathrm{l}$ of trichloroacetate, mixed on vortex type mixer and centrifuged immediately $\left(4^{\circ} \mathrm{C}, 15 \mathrm{~min}, 10000 \mathrm{rpm}\right)$ to remove precipitated protein. The supernatant was purified with a $0.22-\mu \mathrm{m}$ filter. We measured amino acid concentrations with an automatic amino acid analyzer (model L-8900; Hitachi) that separates amino acids by cation-exchange chromatography. Each amino acid was detected spectrophotometrically after a post-column reaction with ninhydrin reagent. Reproducibility was verified by measurements of an amino acid mixture standard solution (Type AN-II; Wako) ${ }^{(14)}$.

\section{Glucose assay to measure glucose content of the medium}

The amount of glucose in the culture medium was measured after IPEC-J2 cells were incubated in the glucose production medium supplemented with different arginine concentrations for $24 \mathrm{~h}$. Glucose was detected using a Glucose Assay Kit (Rsbio), according to the manufacturer's instructions.

\section{Statistical analysis}

Arithmetic means with their standard errors are presented together. Statistical analyses were performed using SPSS Statistics 17.0 (IBM SPSS). The data were analysed using one-way ANOVA and were given as the mean values. Significance was determined by $* P<0.05$ and $* * * 0<01$. 


\section{Results}

\section{Effects of arginine concentrations on small intestinal epithelial cell line integrity}

Fig. 1(a) shows the cell culture schematic diagram. The TEER value of IPEC-J2 cells dropped significantly when arginine was removed from the cultural medium (Fig. 1(b)). However, the TEER in 0.2 and $0.7 \mathrm{~mm}$-arginine groups was maintained at high values. Moreover, the claudin-1,occludin and E-cadherin are important component proteins of TJ, which control the intestinal epithelium paracellular pathway ${ }^{(15,16)}$. Compared with $0.7 \mathrm{~mm}$-arginine, treatment with $0.2 \mathrm{~mm}$-arginine in the medium did not affect the protein expression levels of claudin-1, occludin and E-cadherin (Fig. 1(c)). However, the p-ERK expression level was also not affected, compared with $0.7 \mathrm{~mm}$-arginine groups (Fig. 1(d)). We also did not detect a significant difference in mucin-1 expression by IPEC-J2 cells in the 0.2 and $0.7 \mathrm{~mm}$-arginine groups (Fig. 1(e)). However, the expression levels of TJ proteins and mucin-1 were all significantly decreased in the arginine-free group (Fig. 1(c) and (e)).

\section{Effects of arginine concentrations on CAT1, EAAT3 and ASCT1 mRNA expression in small intestinal epithelial cell line}

The lumenal plasma membrane of absorptive cells contains at least three amino acid transporters, one each for acidic, basic and neutral amino acids ${ }^{(17)}$. Compared with $0.7 \mathrm{~mm}$-arginine in the medium, reducing the arginine concentration in the cell culture medium enhanced the mRNA expression levels of amino acid transporters. We found that the abundance of CAT1, EAAT3 and ASCT1 mRNA expression after treatment with $0.2 \mathrm{~mm}$-arginine increased significantly when compared with the other two groups (Fig. 2).

\section{Effects of arginine concentrations on amino acid uptake by small intestinal epithelial cell line}

The uptake of the basic amino acids (lysine and histidine) by IPEC-J2 cells treated with $0.2 \mathrm{~mm}$-arginine was increased significantly at 12 and $24 \mathrm{~h}$ when compared with IPEC-J2 cells treated with $0.7 \mathrm{~mm}$. However, the uptake of lysine, histidine and arginine IPEC-J2 cells in arginine-free medium was decreased significantly compared with the other two groups (Fig. 3(a) and (b)). We also found that the uptake of the neutral amino acid threonine was also increased under $0.2 \mathrm{~mm}$-arginine in the medium (Fig. 3(c) and (d)). Our results revealed that the uptake of the acidic amino acid (aspartic acid) by IPEC-J2 cells was significantly decreased in response to arginine at concentrations ranging from 0.7 to $0 \mathrm{~mm}$ at 12 and $24 \mathrm{~h}$ (Fig. 3(e) and (f)). (a)

Culture system
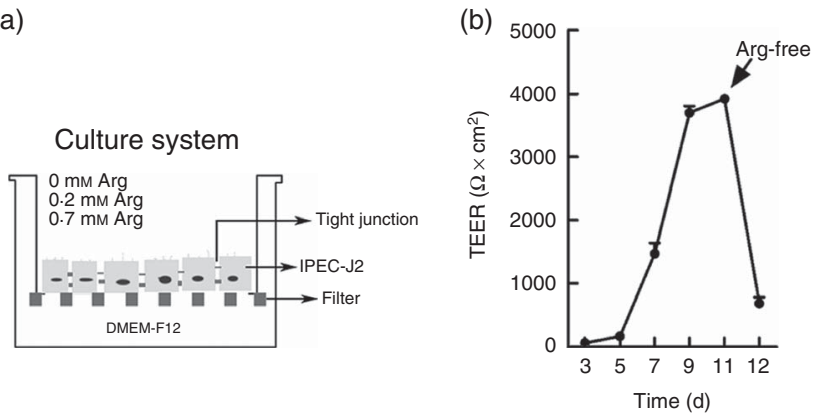

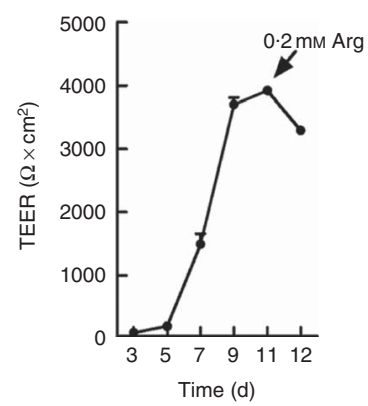

(c)

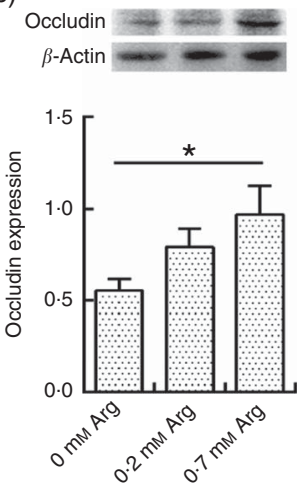

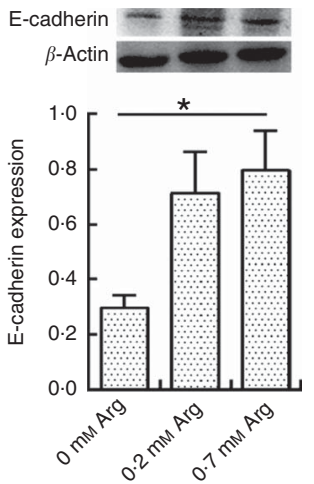
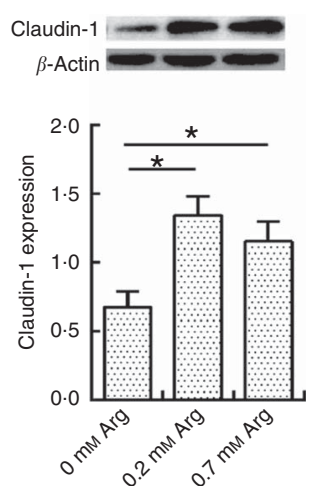

(d)

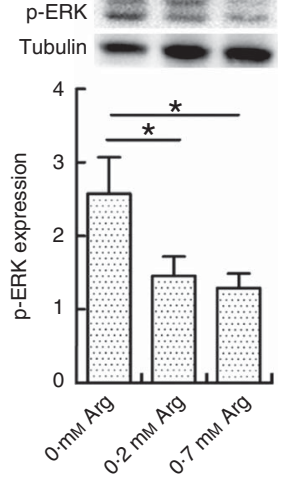

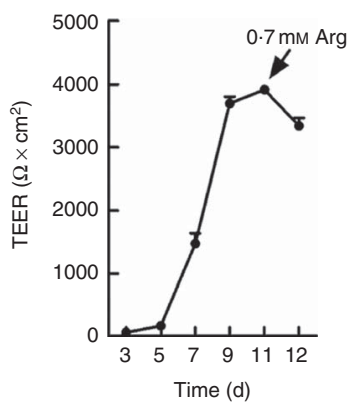

(e)

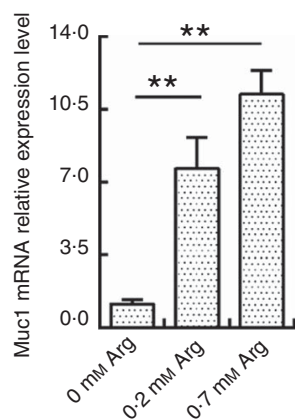

Fig. 1. Effects of arginine levels on small intestinal epithelial cell line (IPEC-J2). (a) Experimental settings to study the barrier function of IPEC-J2 cells in a culture system. The schematic depicts the monoculture system, where IPEC-J2 cells were cultured with Dulbecco's modified Eagle's medium/nutrient mixture F-12 (DMEM-F12). When the IPEC-J2 cells reached confluence, they were cultured in new medium supplemented with different arginine concentrations (arginine-free, 0.2 or $0.7 \mathrm{~mm}$-arginine). (b) Transepithelial electrical resistance (TEER) of arginine-free, $0.2 \mathrm{~mm}$-arginine or $0.7 \mathrm{~mm}$-arginine-cultured IPEC-J2 cells from 3 rd to 12 th day after media replacement. (c) The protein expression levels of occludin, E-cadherin and claudin-1 were detected by Western blot analysis. (d) The protein expression levels of phosphorylated extracellular signal-regulated protein kinases (p-ERK) were also detected by Western blot analysis. (e) Mucin-1 (Muc1) mRNA expression was detected by real-time PCR. Values are means $(n)$, with their standard errors. Results are from four different experiments. ${ }^{*} P<0.05$ and ${ }^{\star *} P<0.01$ as determined by ANOVA. 
(a)

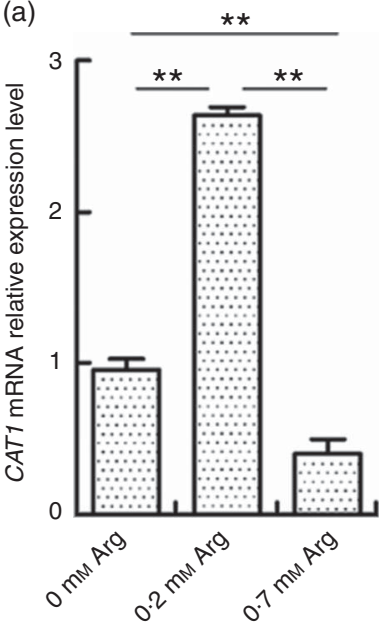

(b)

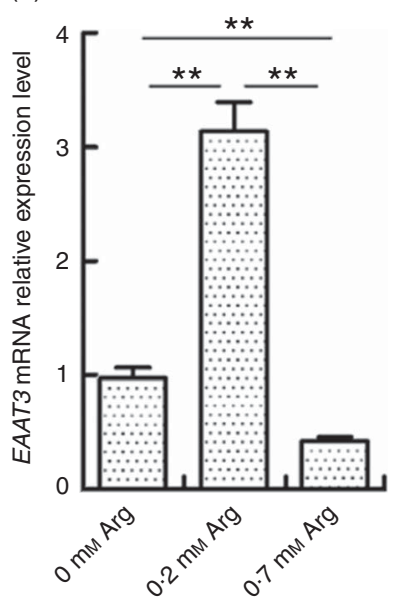

(c)

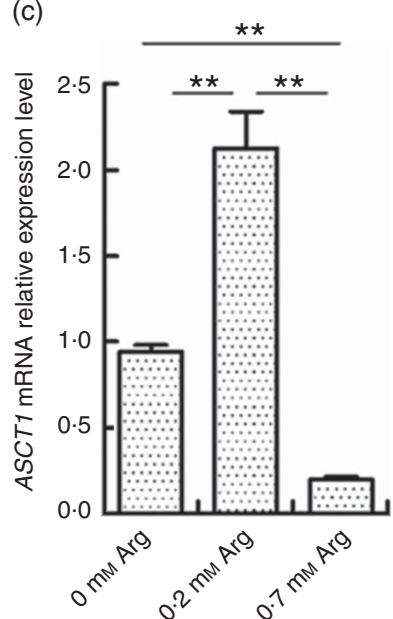

Fig. 2. Effects of arginine levels on CAT1, EAAT3 and ASCT1 expression in small intestinal epithelial cell line (IPEC-J2). Cells were cultured for 24 $\mathrm{h}$ in arginine-free Dulbecco's modified Eagle's medium (DMEM) containing 0.2 or 0.7 mm-arginine. CAT1 (a), EAAT3 (b) and ASCT1 (c) mRNA expression levels were detected by real-time PCR. Values are means $(n 4)$, with their standard errors. Results are from four different experiments. ${ }^{\star *} P<0.01$ as determined by ANOVA.

(a)

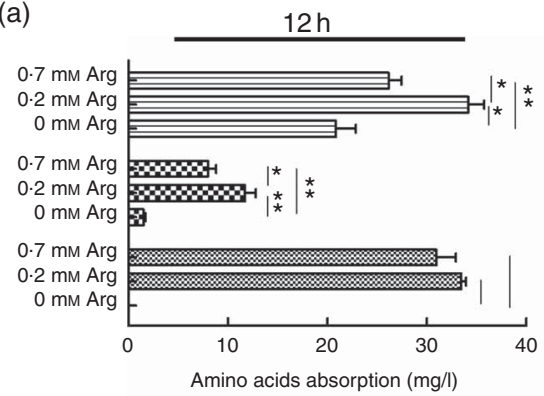

(c)

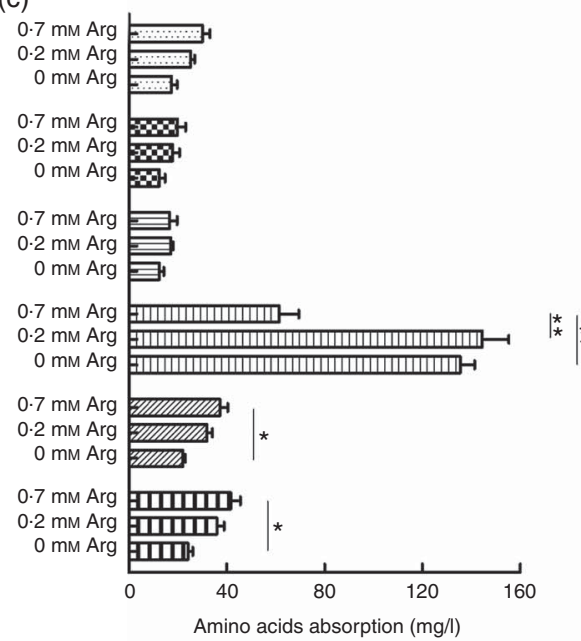

(e)

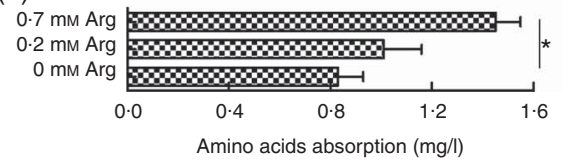

(b)

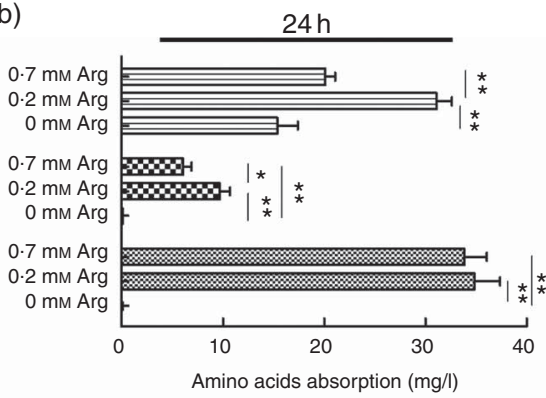

(d)

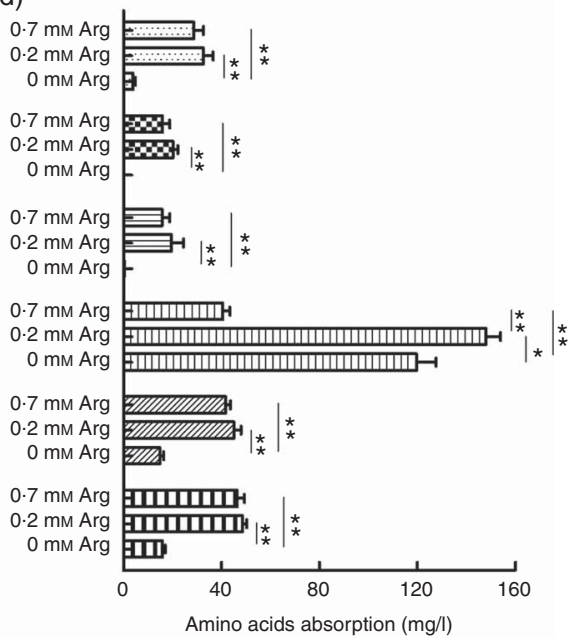

(f)

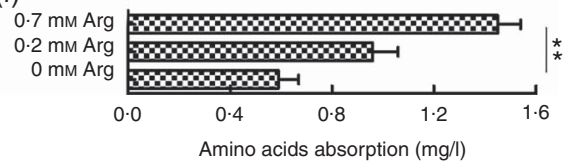

Fig. 3. The effect of arginine levels on amino acid uptake by small intestinal epithelial cell line (IPEC-J2). Cells were cultured in Dulbecco's modified Eagle's medium (DMEM) without arginine, or containing $0.2 \mathrm{~mm}$-arginine or $0.7 \mathrm{~mm}$-arginine for 12 or $24 \mathrm{~h}$, respectively. The level of absorption of basic amino acid (目, lysine; 圈, histidine

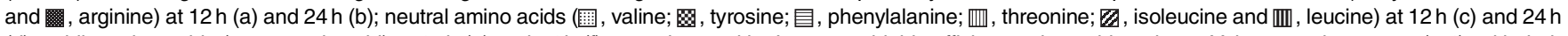
(d); acidic amino acids (图, aspartic acid) at $12 \mathrm{~h} \mathrm{(e)} \mathrm{and} 24 \mathrm{~h}(\mathrm{f})$ were detected by L-8900, a highly efficient amino acid analyzer. Values are the means ( $\mathrm{n} 4$ ), with their standard errors. Results are from four different experiments ${ }^{*} P<0.05$ and ${ }^{* \star} P<0.01$ as determined by ANOVA. 
(a)
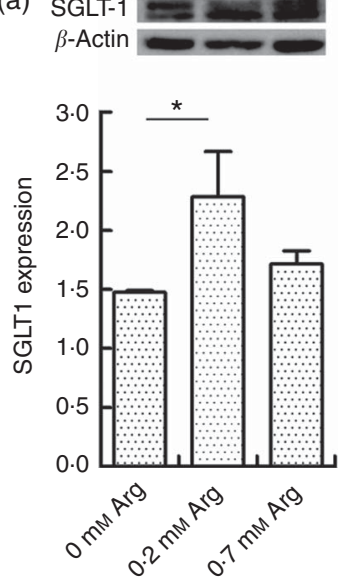

(c)
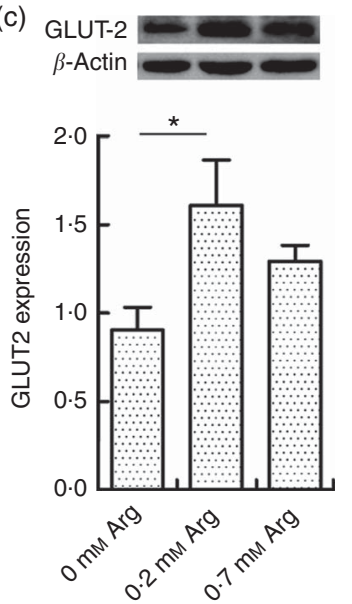

(b)

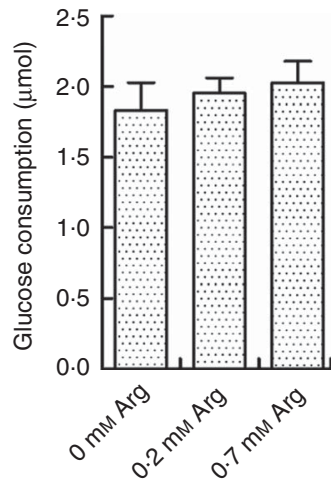

(d)

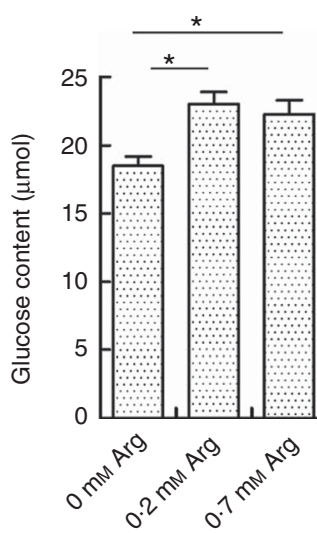

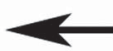

Apical membrane

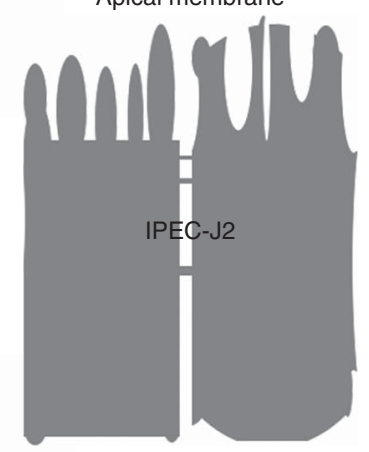

Basement membrance

Fig. 4. Effects of arginine levels on glucose transport by small intestinal epithelial cell line (IPEC-J2). Cells were cultured in Dulbecco's modified Eagle's medium (DMEM) without arginine, or containing, $0.2 \mathrm{~mm}$-arginine or $0.7 \mathrm{~mm}$-arginine for 12 or $24 \mathrm{~h}$, respectively. Sodium/glucose cotransporter 1 (SGLT1) (a) and glucose transporter type 2 (GLUT2) (c) protein expression was detected by Western blot analysis. Glucose transport levels were calculated from the glucose concentration difference between the apical (b) and basolateral (d) sides of the IPEC-J2 cells using a Glucose Assay Kit. Values are the means $(n 4)$, with their standard errors. Results are from four different experiments. ${ }^{*} P<0.05$ as determined by ANOVA.

\section{Effects of arginine on glucose transport in small intestinal epithelial cell line}

Intestinal glucose absorption is mediated by the Na-dependent GLUT SGLT1, and the facilitated glucose transporter GLUT2 provides a glucose exit into the bloodstream at the basolateral side $^{(18)}$. Our results showed that compared with the $0 \mathrm{~mm}$ arginine group the $0.2 \mathrm{~mm}$-arginine group had significantly increased SGLT1 and GLUT2 expression in the IPEC-J2 cells (Fig. 4(a) and (c)). However, there was no significant difference in $\mathrm{Na}^{+}$-dependent glucose absorption capacity among the three groups (Fig. 4(b)). Cells treated with $0.2 \mathrm{~mm}$-arginine could maintain the glucose concentration in the basolateral side, compared with cells treated with $0.7 \mathrm{~mm}$-arginine. However, the glucose content at the basolateral surface of IPEC-J2 cells treated with arginine-free medium was significantly decreased compared with the other two groups (Fig. 4(d)).

\section{Discussion}

Arginine stimulates protein synthesis and enhances cell survival ${ }^{(11)}$. In the intestine, arginine could protect the mucosal barrier by reducing intestinal permeability and improving intestinal structure and function ${ }^{(19,20)}$. In the present study, we found that arginine is necessary for maintaining the mucosal barrier, which was demonstrated by the TEER value dropping rapidly in arginine-free medium. However, 0.2 and $0.7 \mathrm{~mm}$-arginine medium could maintain similar TEER values. This phenomenon is consistent to the previous findings in IPEC-1 cells, which showed that the number of cells and protein synthesis did not differ between the 350 and $500 \mu \mathrm{m}$-arginine-treated groups $^{(21)}$

TJ and adherens junction are important in the maintenance of epithelial integrity. Claudin-1 and occludin protein are two important components of TJ, whereas E-cadherin is the major transmembrane protein of the adherens junction ${ }^{(22,23)}$. Formation of the adherens junction leads to assembly of the $\mathrm{TJ}^{(24)}$. The function of arginine in cell integrity was further verified by claudin-1 and mucin-1 expression, both of which were decreased markedly in arginine-free medium. Compared with the $0.7 \mathrm{~mm}$-arginine medium, a similar trend of claudin- 1 and mucin-1 expressions was detected in cells treated with the $0 \cdot 2 \mathrm{~mm}$-arginine medium. The activation of p-ERK will disrupt 
the TJ structure ${ }^{(25,26)}$. We found that the p-ERK expression level in cells treated with $0.2 \mathrm{~mm}$-arginine in the medium was maintained at normal level, compared with the $0.7 \mathrm{~mm}$ group. These results together demonstrated that arginine is necessary for the maintenance of IPEC-J2 cell integrity, and reducing arginine concentration from 0.7 to $0.2 \mathrm{~mm}$ did not affect cell integrity.

Amino acids are indispensable for epithelium survival and its biological functions. Amino acids are efficiently absorbed by small intestinal enterocytes through amino acid transporters ${ }^{(27)}$. The transporters for amino acids in the small intestine can be divided into basic, neutral and acidic amino acid transporter systems $^{(28)}$. Arginine could stimulate CAT-1-mediated arginine uptake and regulation of inducible nitric oxide synthase for growth, as observed in chick intestinal epithelial cells ${ }^{(29)}$. In this experiment, we found that reducing arginine concentration to $0.2 \mathrm{~mm}$ could stimulate greater expression of Slc7A1 (CAT1), Slc1A1 (EAAT3) and Slc1A4 (ASCT1) mRNA when compared with the $0.7 \mathrm{~mm}$-arginine group or without arginine in the medium. The enhanced expression of amino acid transporters implied efficient uptake of amino acids. This was further verified by the amino acid absorption in our experiments. We found that the $0.2 \mathrm{~mm}$-arginine group improved the absorptive capacity of IPEC-J2 cells for essential and functional amino acids (lysine and histidine) at 12 or $24 \mathrm{~h}$, which were mediated by CAT1. Although the absorption amount of arginine in $0.2 \mathrm{~mm}$ medium was not increased significantly and maintained at the same level with $0.7 \mathrm{~mm}$ medium, the arginine absorption efficiency was actually increased, considering the low arginine concentration in $0.2 \mathrm{~mm}$ medium. Previous studies have shown that threonine promotes mucin synthesis and augments intestinal structure ${ }^{(30)}$. The increased threonine absorption in $0.2 \mathrm{~mm}$-arginine medium in this study could explain the maintenance of the TJ protein and mucin-1 mRNA expression levels. Previous findings demonstrated that dietary arginine supplementation could affect the absorption of other amino acids in the small intestinal mucosa ${ }^{(20)}$. In addition, branched-chain amino acids (valine, isoleucine and leucine) may be necessary to maintain normal intestinal development and absorption of amino acids ${ }^{(31,32)}$. We found that the absorption of other detected amino acids (valine, tyrosine, phenylalanine, isoleucine, leucine and aspartic acid) in cells treated with $0.2 \mathrm{~mm}$-arginine could also be maintained at normal levels compared with $0.7 \mathrm{~mm}$-arginine in the medium, which is necessary to protect the epithelial integrity. Under low arginine levels, high absorption efficiency may be a compensatory mechanism for amino acid uptake by IPEC-J2 cells to guarantee normal physiological functions.

Glucose is a major source of energy for body tissues, particularly for the small intestine in young animals ${ }^{(33)}$. Increased amino acid absorption, supported by the high amino acid transporter expression, guarantees energy needs. We found that glucose consumption at the free surface and glucose content at the basolateral surface of IPEC-J2 cells treated with $0.2 \mathrm{~mm}-$ arginine medium were similar to cells treated with the $0.7 \mathrm{~mm}$ arginine medium. Moreover, the protein expression of SGLT1 and GLUT2 at $0 \cdot 2 \mathrm{~mm}$-arginine did not change when compared with $0.7 \mathrm{~mm}$-arginine, which is inconsistent with glucose consumption. However, the expression levels of the SGLT1 and
GLUT2 proteins were decreased significantly in the argininefree medium. One possible reason is that the appropriate arginine dose for promoting cellular function (e.g. barrier development and absorption of amino acids) requires more energy consumption. This needs to be further studied. Previous studies have shown that amino acid and glucose transport could be modulated by $\mathrm{NO}^{(34)}$. Interestingly, arginine is an essential substrate for synthesis of NO. This may partly explain how different arginine concentrations affect the transport of glucose in epithelial cells.

In conclusion, we demonstrated that arginine is necessary for IPEC-J2 cells to maintain the epithelial integrity. However, reducing the arginine concentration from 0.7 to $0.2 \mathrm{~mm}$ did not affect cellular integrity, as measured by TEER, or claudin-1 and mucin-1 expression. This maintenance of cell integrity was also further verified by the stable p-ERK expression. Moreover, $0 \cdot 2$ mm-arginine stimulated amino acid absorption efficiency by increasing amino acid transporter expression, which guarantees a sufficient energy supply with stable SGLT1 and GLUT2 protein expression. This experiment illustrated that reducing arginine concentration to $0.2 \mathrm{~mm}$ is feasible in IPEC-J2 cells, which provides guidance in developing dietary formulas for pigs at low protein concentrations.

\section{Acknowledgements}

M. X. and L. Y.: study conception and design, performance of the experiments, data analysis and interpretation, manuscript writing; Q. H.: performance of the experiments, data analysis; Q. Y.: study conception and design, financial support, administrative support, data analysis and interpretation, manuscript writing, final approval of the manuscript.

This work was supported by the National Basic Research Program of China (973 program 2013CB127302), National Natural Science Foundation of China (31502024), A Project Funded by the Priority Academic Program Development of Jiangsu Higher Education Institutions and State Key Laboratory of Animal Nutrition Fund of China (2004DA125184F1410).

There were no conflicts of interest (financial, professional or personal) relevant to the manuscript.

\section{References}

1. Peterson LW \& Artis D (2014) Intestinal epithelial cells: regulators of barrier function and immune homeostasis. Nat Rev Immunol 14, 141-153.

2. Turner JR (2006) Molecular basis of epithelial barrier regulation: from basic mechanisms to clinical application. $A m J$ Pathol 169, 1901-1909.

3. Rhodes JM (1989) Colonic mucus and mucosal glycoproteins: the key to colitis and cancer? Gut 30, 1660-1666.

4. Liu FN, Li GH, Wen K, et al. (2010) Porcine small intestinal epithelial cell line (IPEC-J2) of Rotavirus infection as a new model for the study of innate immune responses to Rotaviruses and probiotics. Viral Immunol 23, 135-149.

5. Wu G (2010) Functional amino acids in growth, reproduction, and health. Adv Nutr 1, 31-37.

6. Wu G (2009) Amino acids: metabolism, functions, and nutrition. Amino Acids 37, 1-17. 
7. Jacobi SK \& Odle J (2012) Nutritional factors influencing intestinal health of the neonate. Adv Nutr 3, 687-696.

8. Albin DM, Wubben JE, Rowlett JM, et al. (2007) Changes in small intestinal nutrient transport and barrier function after lipopolysaccharide exposure in two pig breeds. J Anim Sci $\mathbf{8 5}$, 2517-2523.

9. Rhoads JM, Chen W, Gookin J, et al. (2004) Arginine stimulates intestinal cell migration through a focal adhesion kinase dependent mechanism. Gut 53, 514-522.

10. Rhoads M, Fu Q, Rippe R, et al. (2004) Focal adhesion kinase (FAK) and p70 s6 kinase are critical for arginine stimulated intestinal cell migration. J Invest Med 52, S291-S292.

11. Bauchart-Thevret C, Cui LW, Wu GY, et al. (2010) Arginineinduced stimulation of protein synthesis and survival in IPEC$\mathrm{J} 2$ cells is mediated by mTOR but not nitric oxide. Am J Physiol Endocrinol Metab 299, E899-E909.

12. Schierack P, Nordhoff M, Pollmann M, et al. (2006) Characterization of a porcine intestinal epithelial cell line for in vitro studies of microbial pathogenesis in swine. Histochem Cell Biol 125, 293-305.

13. Shatos MA, Doherty JM, Orfeo T, et al. (1992) Modulation of the fibrinolytic response of cultured human vascular endothelium by extracellularly generated oxygen radicals. $J$ Biol Chem 267, 597-601.

14. Agostoni C, Carratu B, Boniglia C, et al. (2000) Free amino acid content in standard infant formulas: comparison with human milk. J Am Coll Nutr 19, 434-438.

15. Tang VW \& Goodenough DA (2003) Paracellular ion channel at the tight junction. Biophys $J$ 84, 1660-1673.

16. Kim YS \& Ho SB (2010) Intestinal goblet cells and mucins in health and disease: recent insights and progress. Curr Gastroenterol Rep 12, 319-330.

17. Xiong X, Yang CB \& Yin YL (2012) [Research progress on intestinal amino acid and amino acid transporters]. Sheng $\mathrm{Li} \mathrm{Ke}$ Xue Jin Zhan [Prog Physiol] 43, 202-206.

18. Roder PV, Geillinger KE, Zietek TS, et al. (2014) The role of SGLT1 and GLUT2 in intestinal glucose transport and sensing. PLOS ONE 9, e89977.

19. Corl BA, Odle J, Niu X, et al. (2008) Arginine activates intestinal $\mathrm{p} 70(\mathrm{~S} 6 \mathrm{k})$ and protein synthesis in piglet rotavirus enteritis. J Nutr 138, 24-29.

20. Wang YX, Zhang LL, Zhou GL, et al. (2012) Dietary L-arginine supplementation improves the intestinal development through increasing mucosal Akt and mammalian target of rapamycin signals in intra-uterine growth retarded piglets. $\mathrm{BrJ}$ Nutr 108, 1371-1381.
21. Tan B, Yin YL, Kong XF, et al. (2010) L-Arginine stimulates proliferation and prevents endotoxin-induced death of intestinal cells. Amino Acids 38, 1227-1235.

22. Gumbiner BM (2005) Regulation of cadherin-mediated adhesion in morphogenesis. Nat Rev Mol Cell Biol 6, 622-634.

23. Cording J, Berg J, Kading N, et al. (2013) In tight junctions, claudins regulate the interactions between occludin, tricellulin and marvel D3, which, inversely, modulate claudin oligomerization. J Cell Sci 126, 554-564.

24. Niessen CM (2007) Tight junctions/adherens junctions: basic structure and function. I Invest Dermatol $\mathbf{1 2 7}$, 2525-2532.

25. Wang Y, Zhang J, Yi XJ, et al. (2004) Activation of ERK1/2 MAP kinase pathway induces tight junction disruption in human corneal epithelial cells. Exp Eye Res 78, 125-136.

26. Gonzalez-Mariscal L, Tapia R \& Chamorro D (2008) Crosstalk of tight junction components with signaling pathways. Biochim Biophys Acta 1778, 729-756.

27. Broer S (2008) Amino acid transport across mammalian intestinal and renal epithelia. Physiol Rev 88, 249-286.

28. Chairoungdua A, Segawa H, Kim JY, et al. (1999) Identification of an amino acid transporter associated with the cystinuria-related type II membrane glycoprotein. J Biol Chem 274, 28845-28848.

29. Yuan C, Zhang XY, He Q, et al. (2015) L-arginine stimulates CAT-1-mediated arginine uptake and regulation of inducible nitric oxide synthase for the growth of chick intestinal epithelial cells. Mol Cell Biochem 399, 229-236.

30. Nichols NL \& Bertolo RF (2008) Luminal threonine concentration acutely affects intestinal mucosal protein and mucin synthesis in piglets. J Nutr 138, 1298-1303.

31. Zhang S, Qiao S, Ren M, et al. (2013) Supplementation with branched-chain amino acids to a low-protein diet regulates intestinal expression of amino acid and peptide transporters in weanling pigs. Amino Acids 45, 1191-1205.

32. Cervantes-Ramirez M, Mendez-Trujillo V, Araiza-Pina BA, et al. (2013) Supplemental leucine and isoleucine affect expression of cationic amino acid transporters and myosin, serum concentration of amino acids, and growth performance of pigs. Genet Mol Res 12, 115-126.

33. Zietek $\mathrm{T} \&$ Daniel H (2015) Intestinal nutrient sensing and blood glucose control. Curr Opin Clin Nutr 18, 381-388.

34. Giovanni E \& Mann DLY (2003) Regulation of amino acid and glucose transporters in endothelial and smooth muscle cells. Physiol Rev 83, 183-252. 